\section{Carbon removal from tile-gap structures with oxygen glow discharges}

Thomas Schwarz-Selinger*, Fabio Genoese, Christian Hopf, Wolfgang Jacob

Max-Planck-Institut für Plasmaphysik, EURATOM Association, Boltzmannstr. 2, 85748 Garching, Germany

\section{Abstract}

The removal of redeposited layers from tile gap structures with ITER-like geometries was investigated with various lowtemperature glow discharges in oxygen. The test structure consists of $19 \mathrm{~mm}$ deep gaps with widths ranging from 0.5 to $4 \mathrm{~mm}$. Erosion rates inside the gaps are reduced compared with a directly exposed witness surface. Smallest erosion is found for the lowest particle energies. Erosion at the bottom of the gap increases linearly with the gap width. Erosion on the side walls drops nearly exponentially with increasing distance from the top. Side wall erosion dominates the total eroded amount for all cases investigated. The total eroded amount integrated over the whole inner gap surface is larger than the amount that would be eroded on a flat surface with an area identical to the gap opening.

PACS: 52.77.Bn, 52.77.Dq, 81.05.Uw, 81.15.Jj

JNM keywords: F0400 First Wall Materials, P0500 Plasma Materials Interaction, R0900 Redeposition, S1300 Surface Effects.

*Corresponding/presenting author e-mail: Thomas.SchwarzSelinger@ipp.mpg.de

\section{INTRODUCTION}

Redeposition of carbon accompanied by co-deposition of hydrogen isotopes is expected to be the dominant tritium retention mechanism in ITER $[1,2]$. Hydrocarbon species released from carbon surfaces by plasma-surface interaction processes may migrate to remote areas and become permanently deposited. Co-deposition occurs where the rate of redeposition exceeds the rate of re-erosion $[1,2]$. In particular in tile gaps and in the gaps of macro-brush structures substantial tritium inventories may build up [3], because such areas are shadowed from direct plasma impact so that re-erosion is negligible. Tritium retention in Next Step Devices and removal techniques were recently reviewed by Counsell et al. [1]. Except for thermo-oxidation, which proofed to be successful also for narrow gaps [4] for most of the presently known cleaning methods [5-9] only data for flat surfaces are available and it is not yet known if they are also efficient on structured surfaces. Therefore, assessing the efficiency of cleaning methods to remove co-deposited layers from tile gap structures is a critical issue for the current ITER design.

Oxygen glow discharge cleaning is one possible candidate to remove redeposited hydrocarbon films [1]. It was shown, e.g., in ASDEX Upgrade that removal is very effective, but limited to surfaces with direct contact to the glow discharge [8] and similar results were obtained in TEXTOR [11]. It is

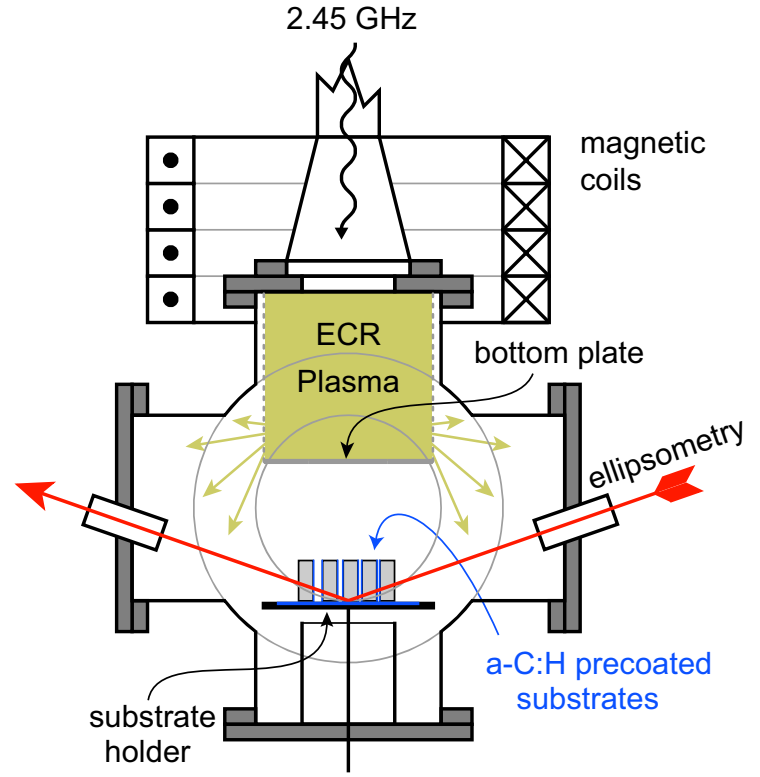

FIG. 1: Schematic representation of the plasma device PLAQ showing the remote ECR configuration where a bottom plate at the plasma cage prevents direct line-of-sight between the plasma and the tile gap test structure, placed at the substrate holder.

known from laboratory experiments, that the ion energy plays a crucial role in the erosion process [12] and that a synergistic interaction between ions and molecular oxygen exists $[10,13]$. One would therefore expect that removal is only effective when particles with substantial energies reach the surface, which is not expected for deep gaps. Recently, Ferreira et al. [14, 15] investigated the removal of hydrocarbon layers from gap structures using dc glow discharges in $\mathrm{He} / \mathrm{O}_{2}$ mixtures. They claim a comparable removal efficiency for films at the bottom of the tile gap compared with the undisturbed surface. However, they used relatively flat gaps (1 mm wide, $3 \mathrm{~mm}$ deep) and did not investigate the removal efficiency on the side walls which have earlier been shown to be the main deposition areas [3].

We investigated the erosion of well characterized hard amorphous hydrogenated carbon $(\mathrm{a}-\mathrm{C}: \mathrm{H})$ films from tile gap test structures with variable aspect ratio. To disentangle the role of neutral species and ions the test structures were exposed to different particle fluxes generated in ECR and rf discharges using pure oxygen as working gas.

\section{EXPERIMENTAL}

The investigated tile gap test structures (TGTS) were exposed to pure oxygen plasmas in the experiment PLAQ depicted schematically in Fig. 1. PLAQ consists of a stainless steel chamber and is equipped with an electron-cyclotronresonance (ECR) plasma source. Details of PLAQ can be found in Ref. [12]. For remote ECR exposure the plasma was 
confined in a metallic cage $(150 \mathrm{~mm}$ in height, $140 \mathrm{~mm}$ in diameter) with a solid metal bottom plate. Particles can leave the cage only through the side wall made of a metal mesh. A substrate holder of $140 \mathrm{~mm}$ in diameter is located $100 \mathrm{~mm}$ below the cage. For direct ECR exposure the bottom plate was removed and a diverging plasma beam impinges perpendicularly on the substrate holder. A capacitively coupled rf plasma (CCP) was produced by applying rf power $(13.56 \mathrm{MHz})$ to the substrate electrode. The ion energy of the species impinging on the TGTS is very different in the three cases. For the remote ECR exposure no energetic ions can reach the TGTS, for direct ECR exposure the ion energy is about $15 \mathrm{eV}$ and for the CCP exposure it is about $300 \mathrm{eV}$. Because the substrate holder is not actively cooled the temperature slightly rises during exposure, depending on the operation conditions. For the remote ECR exposure the temperature never exceeded $300 \mathrm{~K}$ while for the $\mathrm{rf}$ exposure the temperature reached $350 \mathrm{~K}$ at the end of the exposure. However, no substantial influence on the removal rates is expected for these temperatures [13]. The power coupled to the discharges was $75 \mathrm{~W}$ for the ECR discharges and $23 \mathrm{~W}$ for the CCP discharge. The chamber pressure was $0.5 \mathrm{~Pa}$ for the ECR and 2.0 Pa for the CCP discharge. PLAQ is further equipped with in-situ ellipsometry operating at $632.8 \mathrm{~nm}$ to measure erosion and deposition rates at the substrate electrode in real time [16].

All relevant surfaces of the TGTS are covered with a-C:H films which were deposited in a separate chamber employing a capacitively coupled rf plasma at $13.56 \mathrm{MHz}$. Complete single crystalline silicon wafers (100 $\mathrm{mm}$ in diameter) were coated using methane as precursor gas at a pressure of $2.0 \mathrm{~Pa}$ and a dc self bias voltage of $-300 \mathrm{~V}$. Under these conditions typical dense a-C:H films with a hydrogen content $(\mathrm{H} /(\mathrm{H}+\mathrm{C}))$ of $30 \%$, refractive index $\hat{n}=2.12-i \cdot 0.08$ and carbon number density of $9 \times 10^{22} \mathrm{~cm}^{-2}$ are produced. The lateral film thickness distribution on each wafer was analyzed after deposition with ellipsometry using a rotating analyzer ellipsometer (Jobin Yvon PZ 2000) equipped with an automated sample stage allowing 2-dimensional scans with a spot size of $10 \times 30 \mu \mathrm{m}$. The film thickness variation across the whole $100 \mathrm{~mm}$ wafer was always below $3 \%$. The average film thickness of the different wafers produced for this study was $155 \pm 5 \mathrm{~nm}$. The samples for the test structure were cut in rectangular pieces $18 \times 32 \mathrm{~mm}^{2}$ in size.

A photograph of the TGTS is shown in Fig. 2. It consists of three copper cuboids $18 \times 32 \times 8 \mathrm{~mm}^{3}$ in size that separate the four pairs of samples. The different gap widths $g$ of 0.5 , 1,2 , and $4 \mathrm{~mm}$ between the a-C:H coated silicon samples are defined by pairs of small copper spacers $\left(g \times 18 \times 4 \mathrm{~mm}^{3}\right)$. The arrangement is mounted in such a way, that the silicon wafers are flush with the top surface of the copper cuboids. The TGTS was put on at the outer rim of an a-C:H coated, $100 \mathrm{~mm} \mathrm{Si}$ wafer placed on the substrate holder in order not to block the ellipsometry spot in the center of the wafer. The thickness change on the Si wafer can thus be recorded in real time during exposure. Exposure times were set in such a way that most of the a-C:H film was eroded on the flat surface far way from the TGTS. This reference position is marked as $y_{0}$ in Fig. 2.

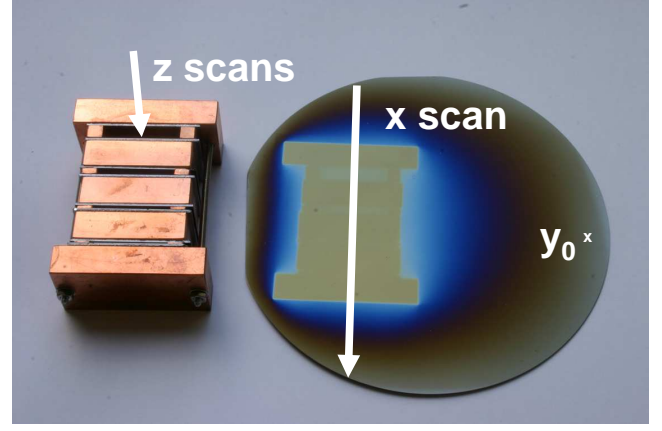

FIG. 2: Picture showing the tile gap test structure next to the bottom silicon wafer after exposure to the remote ECR plasma. Interference colors visible on the bottom wafer indicate the inhomogeneous erosion. Scan directions for the ex situ analyses are indicated with arrows. $y_{0}$ is the reference position for comparison with the erosion on a flat surface.

After exposure the structure was disassembled and analyzed with ellipsometry using a step width of $0.1 \mathrm{~mm}$. The thickness variation was measured in the middle of the side wall samples along the penetration depth as well as on the bottom wafer underneath the test structure ( $x$ scan and $z$ scan in Fig. 2). In addition, scans perpendicular to the $z$ scans were performed for the side wall samples. Because the spacers between the side wall samples cover the original film during exposure they locally prevent erosion. The resulting step edge between the eroded area and the initial film thickness can be determined with an accuracy of $0.1 \mathrm{~nm}$.

\section{RESULTS}

Fig. 2 shows a photograph of the TGTS next to the bottom wafer after exposure to the remote ECR plasma for $96 \mathrm{~h}$. Before exposure the a-C:H film thickness on the silicon wafer was constant and no interference colors were visible. After exposure one can clearly see interference colors, indicating that significant inhomogeneous erosion took place. The initial color of the wafer can still be seen in the area where the TGTS has covered the wafer. Minor color changes can be seen underneath the $4 \mathrm{~mm}$ and $2 \mathrm{~mm}$ gap indicating that erosion is drastically reduced compared with the surface far away from the TGTS.

Fig. 3 depicts the erosion profile of the bottom wafer shown in Fig. 2 ( $x$ scan) as determined by ellipsometry. Only very little erosion took place at the bottom of the gaps compared to the flat surface. Less than $8 \mathrm{~nm}$ are removed in the $4 \mathrm{~mm}$ gap and only $1 \mathrm{~nm}$ is removed in the $0.5 \mathrm{~mm}$ gap. A scan perpendicular to $\mathrm{x}$ shows that erosion outside the TGTS is much stronger and increases with increasing distance from the TGTS. The gradient is steep close to the TGTS (see Fig. 3 around 6 and $56 \mathrm{~mm}$ ), but levels off for distances larger than about $3 \mathrm{~cm}$. We assume that the difference in erosion around the TGTS is due to geometrical shadowing of the reactive par- 


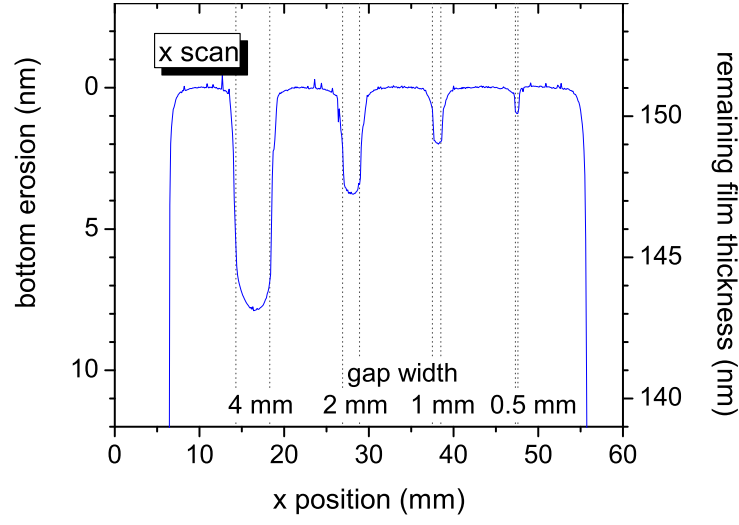

FIG. 3: Bottom erosion in $\mathrm{x}$ direction after exposure to the remote ECR plasma. The original a-C:H film thickness was $151 \mathrm{~nm}$. The remaining film thickness is shown on the right-hand scale.

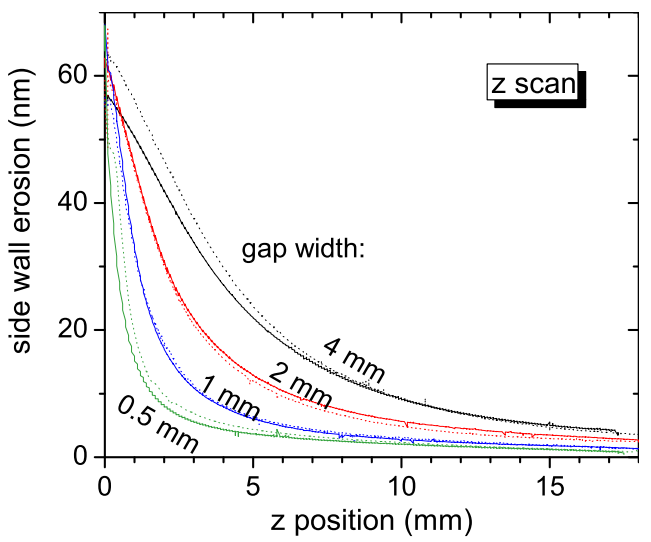

FIG. 4: Side wall erosion in $\mathrm{z}$ direction after exposure to the remote ECR plasma (solid lines = left-hand side, dotted lines = right-hand side).

ticle flux by the TGTS. Maximum erosion of $120 \mathrm{~nm}$ is found in position $y_{0}$ at the outer rim of the wafer. This position can be considered as representative for the erosion on an undisturbed flat witness surface.

In order to compare the results for the three different plasma exposure conditions we normalize the values of the bottom erosion in the TGTS to the erosion of the flat surface at position $y_{0}$ (see Fig. 2). It turns out, that bottom erosion is least effective for the remote exposure and most effective for the CCP case. Bottom erosion increases linearly with gap width from about $0.8 \%$ to $6 \%$ for the remote ECR and from about $1 \%$ to $14 \%$ for the direct ECR cases. For the CCP exposure we find rather constant erosion for the $4 \mathrm{~mm}$ and $2 \mathrm{~mm}$ gaps $(\approx 60 \%)$ and reduced erosion for the smaller gaps $(34 \%$ and $17 \%$ ). One explanation for the last observation could be that for the wider gaps the CCP plasma can penetrate inside while for the smaller ones it cannot.

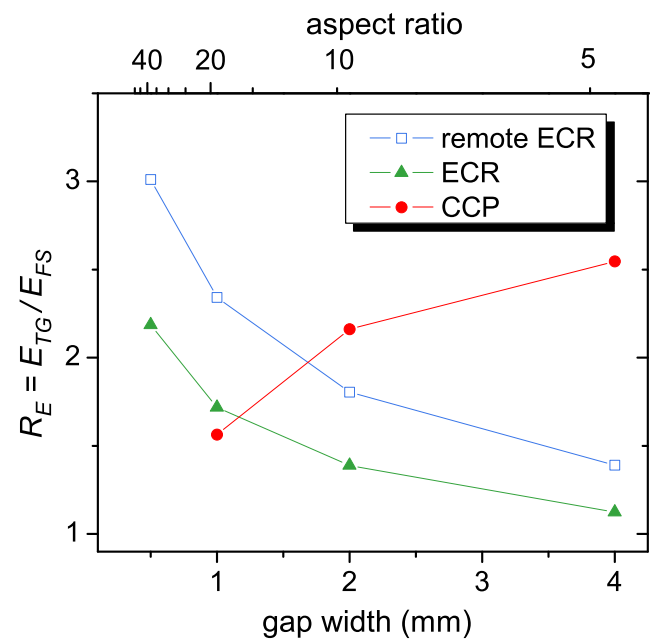

FIG. 5: Plot of the ratio $R_{E}=E_{T G} / E_{F S}$ of the total amount of eroded material inside the TGTS compared with the eroded amount on a flat surface with identical surface area as the opening of the gap for the different investigated plasma conditions.

Fig. 4 shows the erosion on the side walls of the TGTS exposed to the remote ECR discharge ( $z$ scan in Fig. 2). For all four pairs of samples about $60 \mathrm{~nm}$ are eroded at the top of the side walls. For all gap widths the erosion drops rapidly with increasing distance from the top. The 1/e decay length of the erosion rate scales roughly as the gap width.

Comparing erosion on the side walls and at the bottom of the TGTS we find that in every investigated case in total more carbon is eroded from the side wall than from the bottom. In the following, we compare the total amount of carbon eroded from all surfaces inside the gap, $E_{T G}$, with the amount eroded on a flat surface, $E_{F S}$, with the same surface area as the opening of the gap. The ratio $R_{E}=E_{T G} / E_{F S}$ for the different cases is presented in Fig. 5. The surprising result is that the total eroded amount inside the TGTS is larger than on the flat surface. Obviously, the conversion of the reactive particle flux towards the surface into volatile erosion species is more efficient inside the gap than compared with the flat surface.

The behavior of $R_{E}$ is different for the ECR and CCP cases. For the ECR plasma and large gap widths (2 and $4 \mathrm{~mm}) R_{E}$ is between 1 and 1.5. For the smaller gap widths it increases up to 3 . In these cases ion bombardment plays a negligible role and neutral species should therefore dominate the erosion process. The higher erosion probability inside the smaller gaps could tentatively be interpreted as an enhancement of the erosion probability of the neutral species due to trapping inside the gap structure which leads to several collisions with the sidewalls and, therefore, several attempts to erode a surface species. On the flat surface the impinging species have only one surface collision. While for the ECR cases $R_{E}$ decreases with increasing gap width, it increases in the CCP case. The increase of erosion efficiency could be explained by an increased contribution of ions to the erosion inside the TGTS. 
The fact that it is larger than on the free surface could be due to a saturation of ion effects on the flat surface. Inside the TGTS the ion flux is distributed over a larger surface area so that the local ion flux density is reduced and saturation is not yet reached. Another possible explanation is that in the CCP case the plasma starts to penetrate into the TGTS for the larger gap widths and thus increases erosion. A clarification of this point requires further experiments.

\section{CONCLUSIONS}

Exposures of tile gap test structures to different low temperature plasma discharges in oxygen clearly show that removal of redeposited carbon layers inside the gaps is possible. The eroded amount integrated over the whole gap surface is larger than the amount eroded on a flat surface. However, the erosion rates are largely reduced compared to flat surfaces. For all cases investigated, erosion at the side walls of the gaps dominates the total eroded amount. Because side wall erosion decays nearly exponentially (with the distance from the top) with a decay length comparable to the gap width, film removal deep inside the gaps is inefficient. Bottom erosion is largely reduced compared to the flat surface, but increases linearly with gap width. Bottom erosion is only effective when particles with substantial energies hit the surface. Deposition patterns in tile gaps exposed to fusion edge plasmas [3] are similar to our erosion patterns. If the decay lengths of deposition and erosion match then oxygen glow discharge cleaning can be a candidate for tritium removal in tile gaps of plasmafacing surfaces.
[1] G. Counsell, P. Coad, C. Grisolia, C. Hopf, W. Jacob, A. Kirschner, A. Kreter, K. Krieger, J. Likonen, V. Philipps, J. Roth, M. Rubel, E. Salancon, A. Semerok, F. Tabares, A. Widdowson, Tritium retention in next step devices and the requirements for mitigation and removal techniques, Plas. Phys. Control. Fusion 48 (2006) B189-B199.

[2] W. Jacob, Redeposition of hydrocarbon layers in fusion devices, J. Nucl. Mater. 337-339 (2005) 839.

[3] K. Krieger, W. Jacob, D. Rudakov, R. Bastasz, G. Federici, A. Litnovsky, H. Maier, V. Rohde, G. Strohmayer, W. West, J. Whaley, C. Wong, The ASDEX Upgrade and DIII-D Teams, Formation of deuteriumcarbon inventories in gaps of plasma facing components, J. Nucl. Mater. 363-365 (2007) 870-876.

[4] I. Tanarro, J. Ferreira, V. Herrero, F. Tabarés, C. GómezAleixandre, Removal of carbon films by oxidation in narrow gaps: thermo-oxidation and plasma-assisted studies, J. Nucl. Mater. this issue.

[5] C. Skinner, N. Bekris, J. P. Coad, C. Gentile, M. Glugla, Tritium removal from JET and TFTR tiles by a scanning laser, J. Nucl. Mater. 313-316 (2003) 496.

[6] K. Gibson, G. Counsell, C. Curran, M. Forrest, M. Kay, K. Watkins, The removal of co-deposited hydrocarbon films from plasma facing components using high-power pulsed flashlamp irradiation, J. Nucl. Mater. 337-339 (2005) 565-569.

[7] A. Widdowson, J. Coad, N. Bekris, G. Counsell, M. Forrest, K. Gibson, D. Hole, J. Likonen, W. Parsons, T. Renvall, M. Rubel, JET-EFDA Contributors, Efficacy of photon cleaning of JET divertor tiles, J. Nucl. Mater. 363-365 (2007) 341-345.

[8] C. Hopf, V. Rohde, W. Jacob, A. Herrmann, R. Neu, J. Roth, ASDEX Upgrade Team, Oxygen glow discharge cleaning in ASDEX Upgrade, J. Nucl. Mater. 363-365 (2007) 882-887.

[9] C. Hopf, W. Jacob, V. Rohde, Oxygen glow discharge cleaning in nuclear fusion devices, J. Nucl. Mater. 374 (2008) 413-421.

[10] C. Hopf, M. Schlüter, W. Jacob, Chemical sputtering of carbon films by argon ions and molecular oxygen at cryogenic temperatures, Appl. Phys. Lett. 90 (2007) 224106 p1-p3.

[11] V. Philipps, G. Sergienko, A. Lyssoivan, H. G. Esser, M. Freisinger, A. Kreter, U. Samm, Removal of carbon layers by oxygen glow discharges in TEXTOR, J. Nucl. Mater. 363365 (2007) 929-932.

[12] B. Landkammer, A. von Keudell, W. Jacob, Erosion of thin hydrogenated carbon films in oxygen, oxygen/hydrogen and water plasmas, J. Nucl. Mater. 264 (1999) 48.

[13] C. Hopf, M. Schlüter, T. Schwarz-Selinger, W. Jacob, Chemical sputtering of carbon films by simultaneous irradiation with argon ions and molecular oxygen, New J. Phys. 10 (2008) 093022.

[14] J. A. Ferreira, F. L. Tabarés, D. Tafalla, Alternative cleaning technique for the removal of carbon deposits, J. Nucl. Mater. 363-365 (2007) 888-892.

[15] J. A. Ferreira, F. L. Tabarés, D. Tafalla, Removal of carbon deposits in narrow gaps by oxygen plasmas at low pressure, $\mathrm{J}$. Vac. Sci. Technol. A 25 (2007) 746-750.

[16] W. Fukarek, A. von Keudell, A novel setup for spectroscopic ellipsometry using an acousto-optic tuneable filter, Rev. Sci. Instrum. 66 (1995) 3545. 